\title{
Multi-scale and Multi-orientation Face Recognition using Voting based Extreme Learning Machine
}

\author{
Anisha Shadi \\ M. Tech Research Scholar, \\ Department of \\ Electronics and Communication, \\ VNS Group of Institutions: \\ Faculty of Engineering, \\ Bhopal, India
}

\author{
Anil Khandelwal \\ Assistant Professor, \\ Department of \\ Electronics and Communication, \\ VNS Group of Institutions: \\ Faculty of Engineering, \\ Bhopal, India
}

\begin{abstract}
In our daily life human can remember many faces and can recognize them irrespective of illumination, aging, obstructions, variation in views. Most of researchers have worked on the problem of face recognition to develop an automatic face recognition system with capabilities to recognize faces as human beings can do. However, in unconstrained situations where a face may be captured in outdoor environmental conditions, while under changing illumination and pose variations Face Recog-nition Techniques fails to work. Here, a new face recognition method is implemented based on Gabor filter and Voting based extreme learning machine, it presents an effective algorithm to pose invariant face recognition called as Multiscale and Multi-orientation face classification using voting based extreme learning machine. In proposed approach, facial features are extracted by applying set of Gabor filters and Local directional Pattern (LDP), then histogram pattern of result is obtained which is subjected to generate distinctive feature vectors and further classified using V-ELM classifier.The application area of Wireless sensor network(WSN) in real time environment are unreliable and inaccessible, leads to degradation of network performance. The major issues of WSN are QoS ,power and it is impossible to access the WSN to change its power capacity. Long -hops transmission i.e. high range communication which provides the QoS with more energy consumption leads to reduction in network lifetime. The paper concentrates on adjustment of power , range and bit rates to attain adaptive topology control(ATC) at physical layer to maintain equivalent QoS. The simulation are carries out by using MIXIM 2.3 framework Omnet++ 4.6. The comparison of QoS for nonATC and ATC is presented and an improvement of 29 percentage was resulted.
\end{abstract}

\section{Keywords}

Gabor filter, Face Recognition, LDP : Local directional Pattern, V-ELM : Voting Based Extreme Learning MachineMIXIM, Power, range and QoS

\section{INTRODUCTION}

Biometric-based recognition for reliable and robust human identification has given more importance in the wide range of commercial sectors and has become an active area for research in computer vision and pattern recognition. The most commonly used biometric identities for recognition are fingerprint, face and iris. Out of these, face recognition has received much attention both from the researchers as well as from the real time developers. The importance of face recognition arises from the fact that the face recognition system is non-intrusive and analogous to human perception and has a higher level of public acceptance.

Researchers long ago during 1980 till 2005 have proposed a numerous of face recognition system but their main efforts are how to improve accuracy of system instead of improving the database used for identification, these system undergoes a performance drop with pose variations, varying illumination and changes in expressions as well.

\section{HUMAN FACE RECOGNITION}

Automatic face recognition can be categorized into the following two major areas: [1]

1. Face identification: The process by which the system identifies the face image by performing a one-to-many search against the entire stored face images.

2. Face verification or authentication: The process by which the system verifies the face image by matching a captured face image against the persons stored face image[2].

The performance of the face identification systems is quantified in terms of percentage of correctly identified faces. The performance of face verification system is measured in terms of False Acceptance Rate (FAR) achieved at False Rejection Rate (FRR) or vice versa[1].

\section{FACE RECOGNITION SYSTEM FRAMEWORK}

The face recognition system framework is as shown in Figure

A. Face Detection

Face detection aims at determining the position of a single face in an image.The recognition system needs prior knowledge about the face to segment the image from the background[7,2]. 


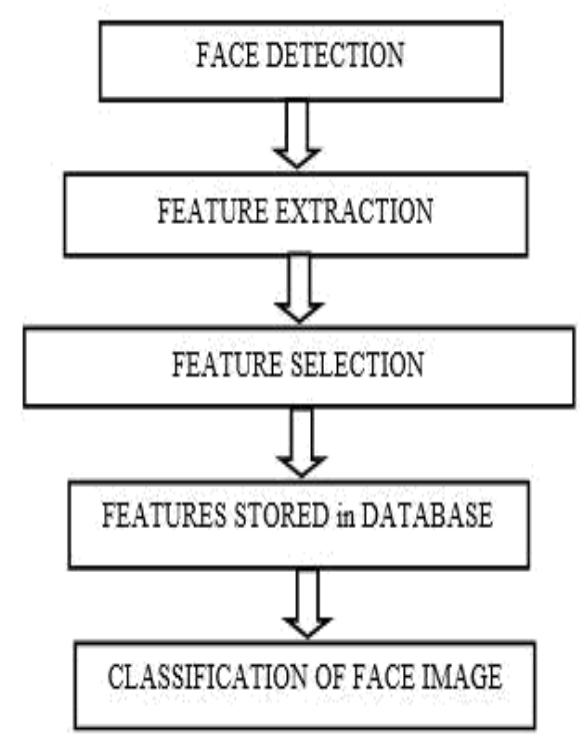

Fig. 1. The face recognition system framework

\section{B. Feature Extraction}

The current feature extraction methods find it difficult to extract the required features because of the wide variability of facial appearance due to the following factors[1].

1. Presence or absence of structural components: Facial features such as beards, moustaches, and glasses may or may not be present.

2. Facial expression: The appearances of faces are directly affected by a persons facial expression[1].

3. Occlusion: Faces may be partially occluded by other objects. In an image with a group of people, some faces may partially occlude other faces which give variation in the extracted features.

4. Image orientation: Face images directly vary for different rotations of the cameras optical axis.

5. Imaging conditions: When the image is formed, factors such as lighting (spectra, source distribution and intensity) and camera characteristics (sensor response and lenses) affect the appearance of a face[7].

6. Pose: The images of a face vary due to the relative camera-face pose (frontal, 45 degree, profile, upside down), and some facial features changes due to the partial or whole occlusion of the image[7,3].

C. Feature Selection

Feature selection is the most important step to improve accuracy and also to reduce training time of our face recognition system. Feature vector generated per image in

our methodology were large enough in dimension and thus it consumes lots of time in generating training dataset. Not all the attributes or features in the image vector are relevant equally. So in order to overcome the curse of dimensionality, the selection of attributes is critically important we need not to include redundant attributes. WEKA 3.6[6] tool have been used for feature selection[5].

D. Classification Model

The performance of the face recognition system mainly depends on the effectiveness of the classification model, thus to improve the accuracy of our face recognition system we have proposed a hybrid model that combines classification technique of Artificial Neural Network (ANN) namely Extreme learning Machine and Gabor Filters[4,2]. This model will solve the issues of requirement of memory to store all the feature vectors and time consumption; there is no need to make assumption regarding the distribution of features. The final stage is based on the information from the classification model, to decide whether the person is a recognized one or not and this model will yields the best estimate of the optimal result.

\section{RELATED WORK VARIOUS FACE RECOGNITION TECHNIQUES AND THEIR REPRESENTATIVE WORK.}

1. Eigenfaces for Recognition: Holistic approach using pixel intensity for feature extraction then dimensionality reduction with Principal component analysis (PCA) and classified using nearest neighbor classifier.[8]

2. ICA-based feature analysis:ICA is better than PCA captures both second and higher order statistics and projects the input data onto the basis vectors that are statistically independent.[9]

3. Two-dimensional PCA a new approach to appearancebased face representation and recognition : This method uses directly $2 \mathrm{D}$ image matrices rather than $1 \mathrm{D}$ vectors for covariance matrix estimation as in PCA, it is mathematically cheap and simple, more suitable for small sample size approaches.[13]

4. Face recognition using Local binary patterns: LBP features are robust to low resolution images. This method is superior to PCA and elastic bunch graph matching. $[11,12]$

5. Low Resolution Single Neural Network Based Face Recognition: It uses single neural network as classifier, which produces straightforward approach towards face recognition. It applies bi-cubic interpolation function on equalized image to get resized image which is lowresolution image thus providing faster processing for training and testing.[13]

6. Component-based face recognition with 3D Morphable models: It is robust, accurate, and easily trainable face recognition system. The component-based system consistently outperformed global face recognition systems in which classication were based on the whole face pattern.[11]

7. Recognition of facial expressions using Gabor wavelets and learning vector quantization: It is observed that LVQ based feature classification technique proposed in this study performs better in recognizing fear expressions than multilayer perceptron (MLP) based classification technique.[14][15].

\section{VARIOUS APPROACHES OF FACE RECOGNITION:[16]}

a) Holistic Approach:

b) Feature-based Approach:

c) Hybrid Approach:

a) Holistic Approach: 
Holistic Approach is an appearance-based methodology which uses holistic texture features and is applied to either complete-face or definite regions in a face image. This approach is based on principal component-analysis (PCA) techniques that can be used to make things easier, a dataset into lower dimension while keeping the characteristics of dataset intact. Subspace analysis is done by extrapolating an image into a lower dimensional subspace generated with the help of training face images and after that recognition is carried out by measuring the distance between known images and the image to be classified. Some well-known face recognition algorithms in this approach for face recognition are Principal Component Analysis (PCA), Independent Component Analysis (ICA), Linear Discriminant Analysis (LDA) and Neural Network Analysis (NNA).

Feature-based Approach: Feature-based methodology uses geometric facial features like mouth, eyes, brows, cheeks etc. and computes geometric relationships between distinct features of face. Complete geometry, dynamic link architecture and hidden Markov model methods fit in to this category. The drawbacks of this system are that it cannot deal with minor inaccuracies of the extracted facial data and it works only with individuals with no face wear like glasses[16, 17].

Hybrid Approach: The idea of hybrid approach comes from how human vision system perceives both local features and the whole face. This approach covers modular Eigen faces[18], hybrid local feature method, shape-normalized and component-based methods[1].

\section{PROPOSED WORK}

Figure2 shows the flowchart for Multiscale and multiorientation face recognition using VELM.

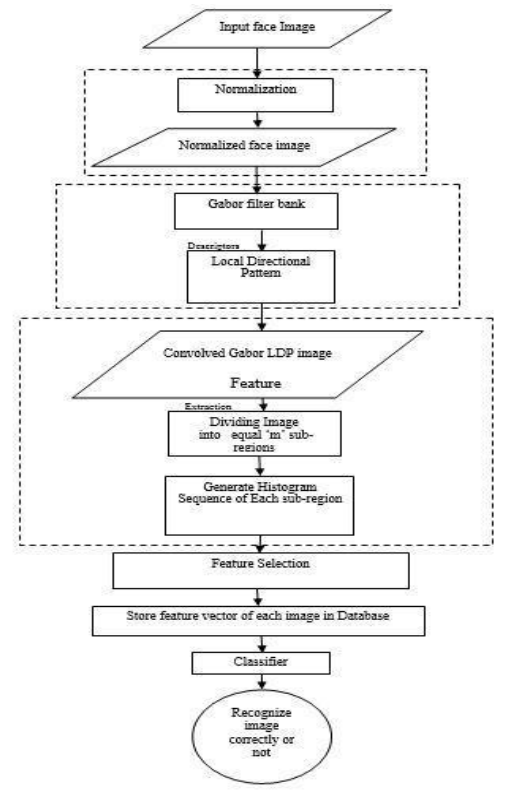

Fig. 2. Multiscale and multiorientation face recognition using VELM.

\section{RESULT ANALYSIS}

The results of face classification using V-ELM classifier can be categorized as True Positive (TP), True Negative (TN), False Positive (FP) and False Negative (FN). The overall accuracy is calculated by the following formula[19].

Overall Accuracy $=(($ Number of $\mathrm{TP}+$ Number of
$\mathrm{TN}) /($ Number of TP+Number of FP+Number of TN+Number of $\mathrm{FN})$ )

\section{Parameter Settings}

The image matrix is normalized first, images taken from a database, after normalization images are applied to the Gabor filter with $0 . .5$ frequencies and 0.8 orientations which gives 40 Gabor filters bank for each image pixel. Thus normalized image is convolved with gabor filters and Gabor images are obtained, which are divided into $3 \times 3$ subregions and LDP is applied to each subregion. After applying LDP to each image subregions then taken mean and variance of the LDP of each subregion of the image thus obtain a row vector of 18 feature elements. As here, LDP is applied to all the 40 Gabor jets, which generates a row/feature vector of 720 elements for each image. To reduce the dimension of large feature vector per images we have applied well known feature selection algorithm inbuilt in WEKA 3.6 tool.

To reduce the dimension of large feature vector per images we have applied well known feature selection algorithm inbuilt in WEKA 3.6 tool.

To optimize feature vector in terms of dimensions we have used WEKA tools inbuilt functionality known as Feature /Attribute Selection,

There are basically two methods for feature selection:[6]

1. Wrapper method

2. Filter method

Wrapper method use a subset evaluator, this evaluator creates all possible subsets from our feature vector. Further it will apply a classification algorithm to induce classifiers from the in each subset. It will select that particular subset of features with which the classification algorithm performs the best. To find an optimized subset the evaluator will use any one of the search technique out of these (random search, breadth first search, depth first search or a hybrid search) and select mode of selection for attribute. There are 2 selection modes cross validation or use full training set.

Filter method uses an attribute evaluator and a ranker to rank all the features in your vector. The number of features we want to select from our vector can be defined. The features that have lower rank are discarded ones and than we check the predictive accuracy of our classification algorithm. Weights put by the ranker algorithms for each feature are different from those by the classification algorithm.

There are numerous of attribute selection algorithm available, we have applied each suitable algorithm as we want to generate output in the form of name of those feature which have been selected in subset. So we applied Bestfit search algorithm for different attribute evaluator.

1. CfsSubsetEval

2. ChiSquaredAttributeEval

3. ConsistencySubsetEval

4. FilteredAttributeEval

5. FilteredSubsetEval

\section{Cfs Subset Eval}

This algorithm evaluates the worth of a subset of attributes or features by considering the individual predictive ability of each feature along with the degree of redundancy between them. Subsets of features that are highly correlated with the 
class while having low inter-correlation are preferred.

\section{Consistency Subset Eval}

This algorithm evaluates the worth of a subset of attributes by the level of consistency in the class values when the training instances are projected onto the subset of attributes. Consistency of any subset can never be lower than that of the full set of attributes, hence the usual practice is to use this subset evaluator in conjunction with a Random or Exhaustive search which looks for the smallest subset with consistency equal to that of the full set of attributes.

\section{Filtered Attribute Evaluator}

Class for running an arbitrary attribute evaluator on data that has been passed through an arbitrary filter (note: filters that alter the order or number of attributes are not allowed). Like the evaluator, the structure of the filter is based exclusively on the training data.

Some of the generated outputs are shown below in Table 1 Based on the subsets obtained by different evaluators, we have analyzed that features obtained around scale value $=0,1$ i.e. parameter in Gabor filter which represents filters scale, are nowhere present in any of the subset obtained from different evaluators which indicates that the features around $=0,1$ are irrelevant in comparison to feature around $=2,3,4$ as they are present in feature selection of best subset at least. Now the optimized feature vector containing approx 430 features per image.

Table I Selected Features And Algorithms Used.

\begin{tabular}{|c|c|}
\hline $\begin{array}{c}\text { FEATURE SELECTION } \\
\text { ALGORITHM }\end{array}$ & SELECTED FEATURES \\
\hline CFS Subset Evaluator & $292,293,294,295,296,29,436,438$ \\
& $474,498,622,628,630,40$ \\
\hline Consistency Subset Evaluator & $292,294,336,438,474,480$ \\
& $498,622,624,628,630,640,64$ \\
\hline Filtered Attribute Evaluator & $292,294,436,438,474,498,622,628,630,640$ \\
\hline
\end{tabular}

Feature vector is applied to train the Neural Network V-ELM. In V-ELM network, 200 input images are used. The V-ELM network architecture used in this experiment consists of 430 input neurons, 10 hidden neurons and 40 output neurons. All the simulated resuts are as shown in Figure 2 (Table 2) and Figure 3 (Table 3).

Table II Performance Evaluation Based On Feature Selection

\begin{tabular}{|c|c|c|c|}
\hline $\begin{array}{c}\text { No of Training } \\
\text { instances } \\
\text { person/class }\end{array}$ & $\begin{array}{c}\text { No of hidden } \\
\text { neurons }\end{array}$ & $\begin{array}{l}\text { Testing } \\
\text { overall } \\
\text { accuracy }\end{array}$ & $\begin{array}{l}\text { Testing } \\
\text { average } \\
\text { accuracy }\end{array}$ \\
\hline 5 & 2 & $97 \%$ & $50 \%$ \\
\hline 5 & 7 & $98 \%$ & $50 \%$ \\
\hline 5 & 8 & $98 \%$ & $50 \%$ \\
\hline 5 & 10 & $99 \%$ & $50 \%$ \\
\hline $\begin{array}{c}\text { No of Training } \\
\text { instances } \\
\text { person/class }\end{array}$ & $\begin{array}{c}\text { No of hidden } \\
\text { neurons }\end{array}$ & $\begin{array}{l}\text { Testing } \\
\text { overall } \\
\text { accuracy }\end{array}$ & $\begin{array}{l}\text { Testing } \\
\text { average } \\
\text { accuracy }\end{array}$ \\
\hline
\end{tabular}

\begin{tabular}{|c|c|c|c|}
2 & 10 & $100 \%$ & $52 \%$ \\
\hline 4 & 10 & $98.75 \%$ & $50 \%$ \\
\hline 5 & 10 & $97.7 \%$ & $50 \%$ \\
\hline 8 & 10 & $98.1 \%$ & $50 \%$ \\
\hline
\end{tabular}

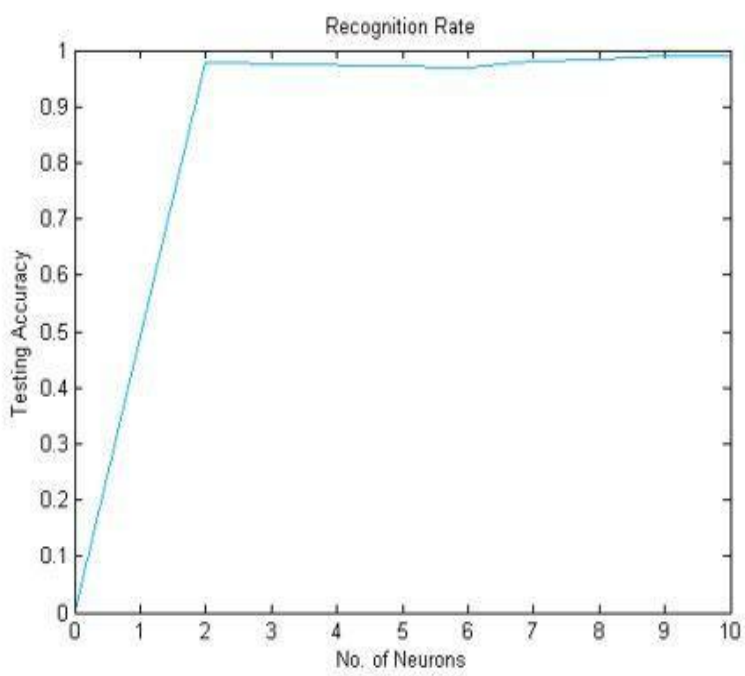

Fig. 3. Recognition Rate using feature selection

Table III Performance Evaluation Without Feature Selection

\begin{tabular}{|c|c|c|c|}
\hline $\begin{array}{c}\text { No of Training } \\
\text { instances } \\
\text { person/class }\end{array}$ & $\begin{array}{c}\text { No of hidden } \\
\text { neurons }\end{array}$ & $\begin{array}{c}\text { Testing } \\
\text { overall } \\
\text { accuracy }\end{array}$ & $\begin{array}{c}\text { Testing } \\
\text { average } \\
\text { accuracy }\end{array}$ \\
\hline 2 & 10 & $91.6 \%$ & $50 \%$ \\
\hline 4 & 10 & $97.75 \%$ & $50 \%$ \\
\hline 5 & 10 & $97.375 \%$ & $50 \%$ \\
\hline 8 & 10 & $98 \%$ & $50 \%$ \\
\hline
\end{tabular}

\section{EXPERIMENT ANALYSIS}

Overall accuracy of proposed face recognition using classifier for ORL database is $99 \%$. In addition to efficient performance, proposed algorithm takes considerably less amount of testing time for ORL database 1.875 seconds which is good enough to achieve real time recognition.

\section{CONCLUSION AND FUTURE DIRECTION}

In this thesis a new face recognition technique Multi-scale and Multi-orientation Face Recognition using voting based extreme learning machine is proposed, this technique gives better performance and accuracy as well. In comparison to various existing face recognition methods proposed algorithm gives reduced training time by using WEKA feature selection functionality for feature vector dimensionality reduction, results in lowering computational complexity and thus less memory requirement to store feature vectors. This algorithm can be used over real time face recognition activities as it takes only 1.875 seconds to classify such a large database containing 400 images.Future efforts will be focused on expression recognition, especially for pose and occlusion variations in unconstrained environment. 


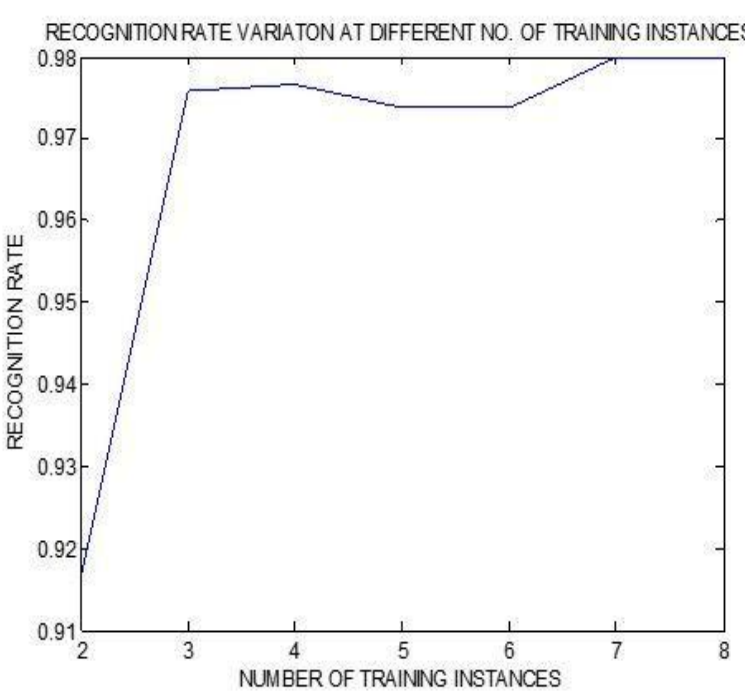

Fig. 4. Recognition Rate without feature selection

\section{REFERENCES}

[1] W. Zhao, R. Chellappa, P.J. Phillips and A. Rosenfeld, Face recogni-tion: A literature survey, ACM Computing Surveys, Vol 35, No. 4,pp. 399458,2003.

[2] C. Sanderson and K. K. Paliwal, Fast features for face authentication under illumination direction changes, Pattern Recogn. Lett., Vol. 24, No. 14, pp. 2409-2419, 2003

[3] Zhang, Ligang Tjondronegoro, Dian Chandran, and Vinod Chandra, "Random Gabor based templates for facial expression recognition in images with facial occlusion",Elsevier, Neurocomputung,Vol 145, 2014.

[4] Suri, P. K.Walia, Ekta Verma and Er. Amit, "Novel face detection using Gabor filter bank with variable threshold", Communication Software and Networks (ICCSN), IEEE 3rd International Conference,pp. 658 661 , 27-29 May 2011.

[5] H. Liu and R. Setiono, A probabilistic approach to feature selection - A filter solution, In: 13th International Conference on Machine Learning, pp.319-327, 1996.

[6] M. A. Hall , Correlation-based Feature Subset Selection for Ma-chine Learning, $\mathrm{PhD}$ thesis, University of Waikato,Hamilton, New Zealand,1999

[7] Guang-Bin Huang, Qin-Yu Zhu and Chee-Kheong Siew, "Extreme Learn-ing Machine: Theory and Application", Neurocomputing, Vol. 70, pp. 489-501, 2006.

[8] M. Turk and A. Pentland, Face Recognition Using Eigenfaces, Proc. IEEE Conf. on Computer Vision and Pattern Recognition, pp. 586-591, 1991.

[9] M.S. Bartlett, J.R. Movellan and T.J. Sejnowski, Face recognition by independent component analysis, IEEE Transaction on Neural Networks, Vol 13,pp. 14501464,2002 .

[10] J. Yang, D. Zhang, A.F. Frangi and J. Yang, Twodimensional PCA: a new approach to appearance-based face representation and recognition, IEEE Transaction on Pattern Analysis ans Machine Intelligence, Vol 26, No.1,pp. $131137,2004$.

[11] TimoAhonen, AbdenourHadid and MattiPietikainen Face Description with Local Binary Patterns: Application to Face Recognition in IEEE Transactions on Pattern Analysis and Machine Intelligence, Vol. 28, No. 12, December 2006

[12] L. Wiskott, J.M. Fellus, N. Kruger and C. VonDerMalsburg, Face recognition by elastic bunch Graph Matching, IEEE Transactions on Pattern Analysis and Machine Intelligence, Vol 19, No. 7, pp. 775779 , 1997.

[13] Z. Jahan, M.Y. Javed and Q. Usman, Low resolution single Neural Network based Face Recognition, Proceedings of the Fourth International Conference on Computer Vision, Image and Signal Processing, Vol. 22, pp. 189193, 2007.

[14] S. Bashyal and G.K. Venayagamoorthy, Recognition of Facial expres-sions using Gabor Wavelets and Learning Vector Quantization, Engineer-ing Applications of Artificial Intelligence, Vol. 21, pp. 10561064, 2008.

[15] De Stefano, C., Sansone, C. and Vento M., Comparing generalization and recognition capability of Learning Vector Quantization and Multilayer Perceptron Architectures, Proceedings of the 9th Scandinavian Confer-ence on Image Analysis, pp. 11231130, June 1995.

[16] L. R. Rama, G. R. Babu and L. Kishore, Face Recognition Based on Eigen Features of Multi Scaled Face Components and Artificial Neural Network, International Journal of Security and Its Applications (IJSIA), Vol. 5, No. 3, pp. 23-44, 2012.

[17] B.S. Manjunath, R. Chellappa and C. von der Malsburg, A Feature based approach to face recognition, Proc. IEEE CS Conf. Computer Vision and Pattern Recognition, pp. 373-378, 1992

[18] A. Pentland, B. Moghaddam and T. Starner, View-Based and modular eigenspaces for face recognition, Proc. IEEE CS Conf. Computer Vision and Pattern Recognition, pp. 84-91, 1994

[19] Sanyam Shukla and R. N. Yadav, "Voting based Extreme Learning Machine with Accuracy based ensemble Pruning" International Journal of Computer Applications (0975 8887), Vol 115 ,No. 22, April 2015. 